\title{
INFLUENCE OF Yb ON VALENCE BAND DENSITY OF STATES OF CdYbTe AND PbYbTe - A RESONANT PHOTOEMISSION STUDY*
}

K. Szamota-Sadowska, B.J. Kowalski, E. Guziewicz, B.A. Orlowski, J. SADowsKi, Z. GolaCKI

Institute of Physics, Polish Academy of Sciences

Al. Lotników 32/46, 02-668 Warszawa, Poland

\section{J. GHiJsen}

Facultes Universitaires Notre-Dame de la Paix Laboratoire Interdisciplinaire de Spectroscopie Electronique 61 rue de Bruxelles, 5000 Namur, Belgium

\section{R.L. JOHNSON}

Universität Hamburg, II Institut für Experimentalphysik Luruper Chaussee 149, 22761 Hamburg 50, Germany
R. BELKHOU,
D. RADOSAVKIČ,
D. Martinotti, N. Barrett
AND C. Guillot

Laboratoire pour l'Utilisation du Rayonnement Électromagnétique, Bat. 209 D

Centre Universitaire Paris-Sud

91405 Orsay Cedex, France

and

DRECAM-SRSIM, CE Saclay, 91191 Gif sur Yvette, France

Photoemission measurements using synchrotron radiation were performed on PbYbTe (bulk crystal) and CdYbTe (MBE thin film). The resonant enhancement of the photoemission was applied for investigation of the contribution of $\mathrm{Yb} 4 f$ electrons to the valence band. The set of the energy distribution curves was collected for energies in the region close to the $4 d-4 f$ Fano transition. The $\mathrm{Yb} 4 f^{14}$ were observed at the binding energies close to the edge of the valence band while the $4 f^{13}$ states were revealed deep in the valence band.

PACS numbers: $61.72 . \mathrm{Vv}$

\footnotetext{
*Supported partially by the Committee for Scientific Research PB No. 2P03B08910.
} 


\section{Introduction}

In this paper we will compare the contribution of $4 f$ electrons to the valence bands of $\mathrm{CdYbTe}$ and $\mathrm{PbYbTe}$ - two tellurides with different crystallographic structures as well as band structures. $\mathrm{PbTe}$ is a narrow-gap semiconductor with a direct gap at the $L$ point, it crystallizes in the rock-salt structure, whereas $\mathrm{CdTe}$ is a wide-gap semiconductor with a direct energy gap at the $\Gamma$ point and with the zinc-blende structure.

Since the resonant behaviour of the photoemission from the $4 f$ shell occurs only for the $\mathrm{Yb}^{3+}\left(4 f^{13}\right)$ state [1], we were able to discriminate between the features connected with $\mathrm{Yb}^{3+}\left(4 f^{13}\right)$ and $\mathrm{Yb}^{2+}\left(4 f^{14}\right)$. Although the resonant photoemission for $\mathrm{Yb}^{2+}\left(4 f^{14}\right)$ does not occur, some changes in the intensity of the structure ascribed to this charge state are clearly observed. These changes are in agreement with the results of a theoretical calculation of the $\mathrm{Yb} 4 f$ photoemission cross-section [2].

\section{Experimental conditions}

In the resonant photoemission experiments we used synchrotron radiation of the photon energies ranging from 30 to $190 \mathrm{eV}$ for $\mathrm{PbYbTe}$ and 90 to $200 \mathrm{eV}$ for CdYbTe.

For PbYbTe the experiments were performed on the unique line Flipper II of DORIS II Storage Ring in HASYLAB, DESY in Hamburg, Germany. The spectra were normalised to the monochromator output and corrected for variations in photons flux.

The spectra of CdYbTe were acquired at the LURE synchrotron radiation laboratory in Orsay, France. The storage ring SUPER-ACO was the source of synchrotron radiation.

The bulk $\mathrm{PbYbTe}$ samples were obtained by a modified Bridgman method in the Institute of Physics, Polish Academy of Sciences in Warsaw. They contained $4 \%$ of $\mathrm{Yb}$. Clean surfaces for the photoemission experiments were prepared in situ by scraping the sample with a diamond file or by cleavage.

The thin film (1.5 $\mu \mathrm{m}$ thick) of CdYbTe was grown by MBE at the growth temperature of $320^{\circ} \mathrm{C}$ on the CdTe (100) buffer layer crystallized on a GaAs (100) substrate $\left(2^{\circ}\right.$ off-oriented towards [0 $\left.\left.0 \overline{1} 1\right]\right)$. The surface of the sample was cleaned by the argon ion bombardment and annealing.

\section{Experimental results and discussion}

Figure 1 presents the energy distribution curves (EDCs) spectra obtained for $\mathrm{PbYbTe}(\mathrm{a})$ and CdYbTe (b). The binding energy is measured with respect to the valence band maximum. The $A$ and $C$ spectra were collected for energies close - to the resonance energy, while $B$ and $D$ spectra were recorded for the energies near to the energy of antiresonance. In order to show the influence of $\mathrm{Yb}$ ions on the valence band structure, in both pictures the differences between the spectra were shown (the lowest spectrum in each figure). The dominant contribution of ytterbium to $\mathrm{PbYbTe}$ valence band was revealed as structures at $-9.4 \mathrm{eV}$ and $-13.6 \mathrm{eV}$. The change in intensity of these peaks as a function of photon energy 

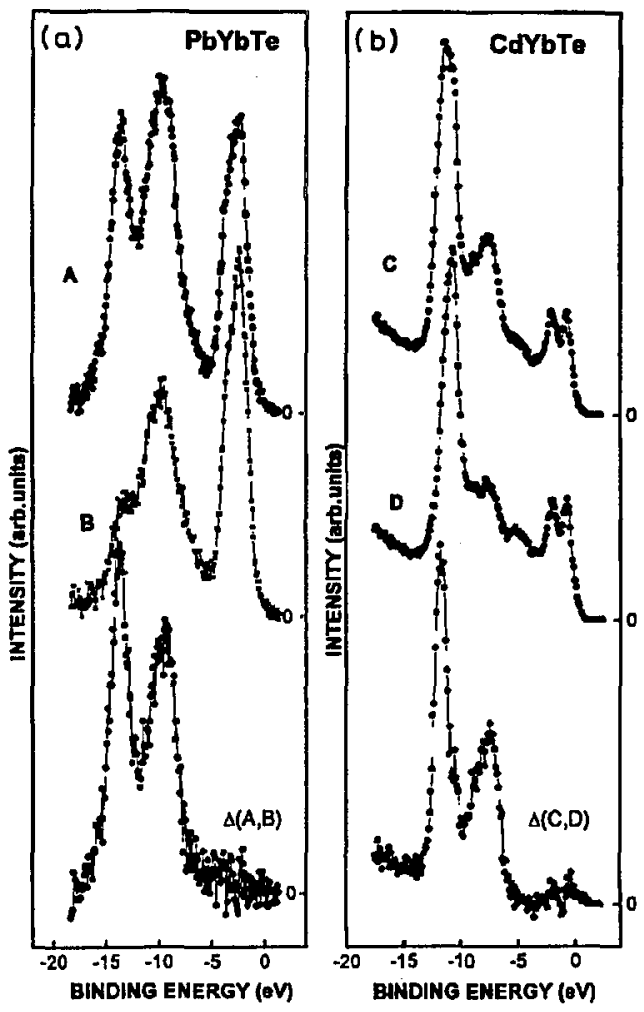

Fig. 1. The sets of EDC spectra of PbYbTe (a) and CdYbTe crystals (b). The photon energies are: $A=184.2 ; B=181.2 ; C=177.5 ; D=174.5 \mathrm{eV}$. The lowest spectrum in each figure shows the differences between the $A$ and $B$ or $C$ and D spectra, respectively. The zero of the energy scale corresponds to the edge of the valence band.

was clearly observed. In the $A$ spectrum these two peaks become higher than in the $B$ spectrum. Taking into account that the resonance occurs only for partly occupied states, we can connect these structures with presence of $4 f^{13}$ state.

The shape of this structure corresponds quite well to the final-state multiplet splitting calculated by Gerken [3]. The energy separation between the peaks is also similar to that obtained for $\mathrm{Yb}_{2} \mathrm{O}_{3}$ [4].

The very similar effect was observed for the curves obtained for the CdYbTe sample. The contribution of $\mathrm{Yb} 4 f^{13}$ was found at $-7.7 \mathrm{eV}$ and $-12 \mathrm{eV}$. The distance between these peaks is very similar to that obtained for $\mathrm{PbYbTe}$.

The set of the energy distribution curves for CdYbTe and CdTe presented in Fig. 2 allows us to compare structure of the valence bands. The spectra for $\mathrm{CdYbTe}$ were recorded for the same photon energies but for surfaces prepared by different procedures. The $F$ spectrum was measured after 30 minutes of $\mathrm{Ar}^{+}$ion sputtering and 35 minutes of heating at $275^{\circ} \mathrm{C}$. The $E$ spectrum was recorded after extra sputtering lasting 20 minutes. In the $E$ curve two maxima appear 


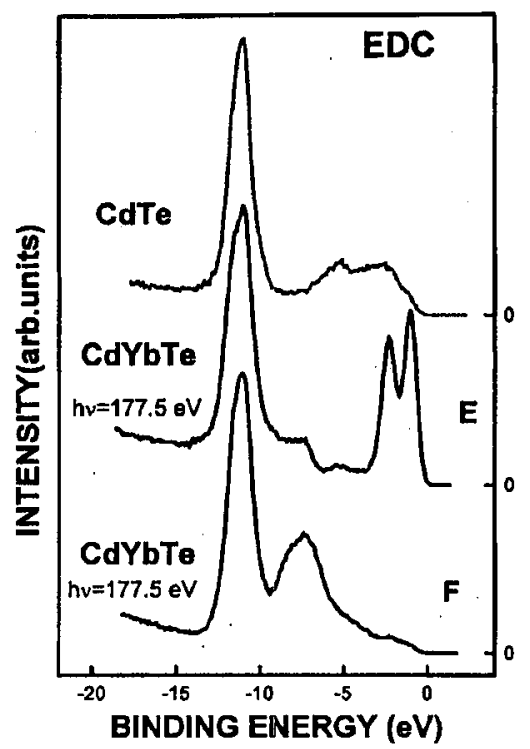

Fig. 2. The EDC spectra for CdTe and CdYbTe. The photon energy has been written above the spectra. The $F$ spectrum was measured after 30 minutes of $\mathrm{Ar}^{+}$ion sputtering and 35 minutes of heating at $275^{\circ} \mathrm{C}$. The $E$ spectrum was recorded after extra sputtering lasting 20 minutes.

at $-1.1 \mathrm{eV}$ and $-2.3 \mathrm{eV}$. The separation between them corresponds well to the spin-orbit splitting of two Yb $4 f^{14}$ components $\left(4 f_{7 / 2}, 4 f_{5 / 2}\right)$ [5]. Thus, the surface preparation procedure can change the charge state of ytterbium ions. More detailed studies show that strength of this feature depends on surface conditions.

\section{Conclusion}

The results of the resonant photoemission studies show that ytterbium introduced as an impurity to $\mathrm{PbTe}$ and $\mathrm{CdTe}$ crystals occurs as $\mathrm{Yb}^{3+}$ ions in both materials. This state was observed as two peaks separated by about $4 \mathrm{eV}$.

After surface treatments of the CdYbTe crystal two maxima characteristic of spin-orbit split components of $\mathrm{Yb}^{2+}$ state appear.

\section{References}

[1] L.C. Davis, L.A. Feldkamp, Phys. Rev. B 23, 6239 (1981).

[2] J.J. Yeh, I. Lindau, At. Data Nucl. Data Tables 32, 1 (1985).

[3] F. Gerken, J. Phys, F 13, 703 (1983).

[4] J. Schmidt-May, F. Gerken, R. Nyholm, L.C. Davis, Phys. Rev. B 30, 5560 (1984).

[5] M. Bodenbach, A. Höhr, C. Laubschat, G. Kaindl, M. Methfessel, Phys. Rev. B 50, 14446 (1994). 